\title{
BIFUNCTORS AND ADJOINT PAIRS
}

\author{
BY \\ J. FISHER PALMQUIST AND DAVID C. NEWELL
}

\begin{abstract}
We use a definition of tensor products of functors to generalize some theorems of homological algebra. We show that adjoint pairs of functors between additive functor categories correspond to bifunctors and that composition of such adjoint pairs corresponds to the tensor product of the bifunctors. We also generalize some homological characterizations of finitely generated projective modules to characterizations of small projectives in a functor category. We apply our results to adjoint pairs arising from satellites and from a functor on the domain categories.
\end{abstract}

0. Introduction. Let $R$ and $S$ be rings, and let $\operatorname{Mod}_{R}$ and $\operatorname{Mod}_{S}$ denote the categories of right $R$-modules and right $S$-modules respectively. Given an $S-R$ bimodule $E$, we obtain a functor $\operatorname{Hom}_{R}(E, \quad): \operatorname{Mod}_{R} \rightarrow \operatorname{Mod}_{S}$ with an adjoint

$\otimes_{S} E: \operatorname{Mod}_{S} \rightarrow \operatorname{Mod}_{R}$, which we shall consider as an adjoint pair

$$
\left(\operatorname{Hom}_{R}(E, \quad), \otimes_{S} E\right): \operatorname{Mod}_{R} \rightarrow \operatorname{Mod}_{S} .
$$

Furthermore, given any adjoint pair $\left(u_{1}, u_{2}\right): \operatorname{Mod}_{R} \rightarrow \operatorname{Mod}_{S}$ (i.e., a functor $u_{1}: \operatorname{Mod}_{R} \rightarrow \operatorname{Mod}_{S}$ having $u_{2}: \operatorname{Mod}_{S} \rightarrow \operatorname{Mod}_{R}$ as an adjoint) it is well known that there is a bimodule $E$ which represents this adjoint pair, that is, one has equivalences $u_{1} \cong \operatorname{Hom}_{R}(E, \quad)$ and $u_{2} \cong \otimes_{S} E$ (see [3] or [6]). In fact, let $E=u_{2}(S)$, then using the fact that $u_{2}$ is right continuous (i.e., $u_{2}$ is right exact and preserves direct sums) one obtains $N \otimes_{S} E=u_{2}(N)$ for every $S$-module $N$ by examining the effect of $u_{2}$ on an $S$-free presentation of $N$. Furthermore, if $T$ is a ring and $\left(v_{1}, v_{2}\right): \operatorname{Mod}_{S} \rightarrow \operatorname{Mod}_{T}$ is an adjoint pair represented by a $T-S$ bimodule $E^{\prime}$, then $\left(v_{1} \circ u_{1}, u_{2} \circ v_{2}\right): \operatorname{Mod}_{R}$ $\rightarrow \operatorname{Mod}_{T}$ is an adjoint pair represented by the $T-R$ bimodule $E^{\prime} \otimes_{S} E$.

Although it is well known that functor categories are generalizations of categories of modules, many of the techniques of homological algebra could not be extended because of a lack of a proper definition of a tensor product. However, in [4], a suitable definition has been given, and in this paper we demonstrate how this definition can be exploited. In particular, we show that the above theorems for modules can be easily extended to functor categories.

Before giving a specific summary of the contents of this paper, we introduce some notation and conventions.

Received by the editors March 29, 1970.

AMS 1969 subject classifications. Primary 1810, 1820.

Key words and phrases. Functor categories, tensor product of functors, bifunctors, adjoint pairs on functor categories, satellites, duality on functors, small projective functors, adjoints of induced functors.

Copyright 두 1971, American Mathematical Society 
We shall assume throughout this paper that all of our categories are at least preadditive and that all of our functors are covariant and additive. If $\mathscr{C}$ and $\mathscr{D}$ are two categories, we shall let $[\mathscr{C}, \mathscr{D}]$ denote the category whose objects are functors from $\mathscr{C}$ to $\mathscr{D}$ and whose morphisms are natural transformations between these functors. Similarly, we let $\left[\mathscr{C}^{\circ}, \mathscr{D}\right]$ denote the category whose objects are contravariant functors from $\mathscr{C}$ to $\mathscr{D}$ (that is, covariant functors from the dual category of $\mathscr{C}, \mathscr{C}^{\circ}$, to $\mathscr{D}$ ). If $F$ and $G$ are objects of [ $\left.\mathscr{C}, \mathscr{D}\right]$, we let $[F, G]$ denote the abelian group of natural transformations from $F$ to $G$. (We shall assume throughout that we are in a proper set-theoretic setting so that all of our categories are well defined.)

Let $\mathrm{Ab}$ denote the category of abelian groups. If $\mathscr{C}$ is a category, then for objects $A$ and $B$ of $\mathscr{C}$ we denote the abelian group of morphisms in $\mathscr{C}$ from $A$ to $B$ by $\mathscr{C}(A, B)$, we let $h_{\mathscr{C}}^{A}=\mathscr{C}(A, \quad)$ (or $h^{A}$ if $\mathscr{C}$ is unambiguous) denote the representable functor from $\mathscr{C}$ to $\mathrm{Ab}$, and similarly we let $h_{B}^{\mathscr{C}}=\mathscr{C}(, B)$, or $h_{B}$, denote the contravariant representable functor from $\mathscr{C}^{\circ}$ to $\mathrm{Ab}$.

In $\S 1$, we define, for functors $F: \mathscr{C} \rightarrow \mathrm{Ab}$ and $G: \mathscr{C}^{\circ} \rightarrow \mathrm{Ab}$, an abelian group $F \otimes_{\mathscr{C}} G$, called the tensor product of $F$ and $G$ over $\mathscr{C}$. This definition and properties (which generalize the properties of the tensor product of modules) have been given in [4], and we present here in a way suitable for applications to this paper a summary of these properties.

If $\mathscr{C}_{1}$ and $\mathscr{C}_{2}$ are categories and $U: \mathscr{C}_{1}^{\circ} \times \mathscr{C}_{2} \rightarrow \mathrm{Ab}$ is a bifunctor, we have a functor $\otimes_{\mathscr{C}_{1}} U:\left[\mathscr{C}_{1}, \mathrm{Ab}\right] \rightarrow\left[\mathscr{C}_{2}, \mathrm{Ab}\right]$ defined as follows: if $F: \mathscr{C}_{1} \rightarrow \mathrm{Ab}$ is a functor and $B$ is an object of $\mathscr{C}_{2}$, then $\left(F \otimes_{\mathscr{C}_{1}} U\right)(B)=F \otimes_{\mathscr{C}_{1}} U(, B)$. We also have a functor $[U,]_{\mathscr{C}_{2}}:\left[\mathscr{C}_{2}, \mathrm{Ab}\right] \rightarrow\left[\mathscr{C}_{1}, \mathrm{Ab}\right]$ defined as follows: for $G: \mathscr{C}_{2} \rightarrow \mathrm{Ab}$ a functor and $A$ and object of $\mathscr{C}_{1}$, we let $[U, G]_{\mathscr{C}_{2}}(A)=[U(A), G$,$] . We complete \S 1$ by showing that $\otimes_{\mathscr{C}_{1}} U$ is adjoint to $[U,]_{\mathscr{C}_{2}}$.

It follows easily from the additive case of a theorem of André (see [1, Proposition 9.1]) that one has an equivalence of categories $\left[\mathscr{C}_{1}^{\circ} \times \mathscr{C}_{2}, \mathrm{Ab}\right] \cong \operatorname{Adj}\left(\left[\mathscr{C}_{2}, \mathrm{Ab}\right],\left[\mathscr{C}_{1}, \mathrm{Ab}\right]\right)$, where the objects of the second category consist of pairs of functors $\left(u_{1}, u_{2}\right), u_{1}:\left[\mathscr{C}_{2}, \mathrm{Ab}\right] \rightarrow\left[\mathscr{C}_{1}, \mathrm{Ab}\right]$ and $u_{2}:\left[\mathscr{C}_{1}, \mathrm{Ab}\right] \rightarrow\left[\mathscr{C}_{2}, \mathrm{Ab}\right]$ its adjoint. In other words, every adjoint pair $\left(u_{1}, u_{2}\right)$ is represented by a bifunctor $U$. We give a simpler proof of this theorem which generalizes the proof mentioned above for the case of modules. Furthermore, if $V: \mathscr{C}_{2}^{\circ} \times \mathscr{C}_{3} \rightarrow \mathrm{Ab}$ is a bifunctor representing an adjoint pair $\left(v_{1}, v_{2}\right):\left[\mathscr{C}_{3}, \mathrm{Ab}\right] \rightarrow\left[\mathscr{C}_{2}, \mathrm{Ab}\right]$, then there is a natural way to define a bifunctor $U \otimes_{\mathscr{C}_{2}} V: \mathscr{C}_{1}^{\circ} \times \mathscr{C}_{3} \rightarrow \mathrm{Ab}$ so that $U \otimes_{\mathscr{C}_{2}} V$ represents the adjoint pair $\left(u_{1} \circ v_{1}\right.$, $\left.v_{2} \circ u_{2}\right)$. These results are given in $\S 2$.

As an example, in $\S 3$ we show that if $F: \mathscr{C} \rightarrow \mathscr{D}$ is a functor between abelian categories and $S^{n} F$ and $S_{n} F$ denote the $n$th right satellite of $F$ and the $n$th left satellite of $F$ respectively, then $S^{n}:[\mathscr{C}, \mathscr{D}] \rightarrow[\mathscr{C}, \mathscr{D}]$ is adjoint to $S_{n}:[\mathscr{C}, \mathscr{D}]$ $\rightarrow[\mathscr{C}, \mathscr{D}]$ (if they exist), and if $\mathscr{D}=\mathrm{Ab}$, the corresponding bifunctor representing this adjoint pair $\left(S_{n}, S^{n}\right)$ is $\operatorname{Ext}^{n}():, \mathscr{C}^{\circ} \times \mathscr{C} \rightarrow \mathrm{Ab}$.

In $\S 4$, we define for a functor $F: \mathscr{C} \rightarrow \mathrm{Ab}$ its "dual" functor $F^{*}: \mathscr{C}^{\circ} \rightarrow \mathrm{Ab}$, 
natural transformations $\otimes_{\mathscr{C}} F^{*} \rightarrow[F$,$] and F \rightarrow F^{* *}$, and we are able to prove the usual theorems about these natural transformations when $F$ is a small projective. We make the easy step of generalizing these results to bifunctors, and as an example, we give a simple description of the adjoint to a functor $u:\left[\mathscr{C}_{2}, \mathrm{Ab}\right]$ $\rightarrow\left[\mathscr{C}_{1}, \mathrm{Ab}\right]$ when $u$ is induced by a functor $f: \mathscr{C}_{1} \rightarrow \mathscr{C}_{2}$.

We note that the results of this paper still hold when $\mathrm{Ab}$ is replaced by Ens, the category of sets, and all of our preadditive categories are replaced by ordinary ones. We do the additive case as the technique involved is more clearly an extention of that of homological algebra.

1. Tensor products of functors. In this section we summarize the properties of the tensor product of functors which are found in [4] in a way convenient for our use in this paper. This summarization is given in the following:

Proposition 1.1. Let $\mathscr{C}$ be a category and let $F: \mathscr{C} \rightarrow \mathrm{Ab}$ and $G: \mathscr{C}^{\circ} \rightarrow \mathrm{Ab}$. Then there is a unique abelian group $F \otimes_{\mathscr{C}} G$ so that the assignment $(F, G) \rightarrow F \otimes_{\mathscr{C}} G$ defines a functor of two variables having the following properties:

(a) For any object $A$ of $\mathscr{C}, h^{A} \otimes_{\mathscr{C}} G \cong G(A)$ and $F \otimes_{\mathscr{C}} h_{A} \cong F(A)$,

(b) $\otimes_{\mathscr{C}} G$, considered as a functor from $[\mathscr{C}, \mathrm{Ab}]$ to $\mathrm{Ab}$, is right continuous, and $F \otimes_{\mathscr{C}}$, considered as a functor from $\left[\mathscr{C}^{\circ}, \mathrm{Ab}\right]$ to $\mathrm{Ab}$, is right continuous.

For the proof we refer to [4]. As a consequence, we have the following:

Proposition 1.2. Let $\mathscr{C}$ be a category, $G: \mathscr{C}^{\circ} \rightarrow \mathrm{Ab}$ and $\nu:[\mathscr{C}, \mathrm{Ab}] \rightarrow \mathrm{Ab}$ be functors, and suppose that for each object $A$ of $\mathscr{C}$ there is a homomorphism $\varphi_{A}^{\prime}: G(A)$ $\rightarrow \nu\left(h^{A}\right)$ which is natural in $A$. Then there is a unique natural transformation $\varphi: \quad \otimes_{\mathscr{C}} G \rightarrow \nu$ such that $\varphi\left(h^{A}\right)$ is the composition of the isomorphism $h^{A} \otimes_{\mathscr{C}} G \simeq G(A)$ followed by $\varphi_{A}^{\prime}$. Furthermore, if $\nu$ is right continuous and $\varphi_{A}^{\prime}$ is an isomorphism of abelian groups for all objects $A$ of $\mathscr{C}$, then $\varphi$ is an isomorphism of functors.

The proof follows from 1.1 and the observation that for any functor $F: \mathscr{C} \rightarrow \mathrm{Ab}$, there are collections of objects $\left(A_{i}\right)_{I}$ and $\left(A_{j}\right)_{J}$ of $\mathscr{C}$ and an exact sequence

$$
\sum_{J} h^{A_{j}} \rightarrow \sum_{I} h^{A_{i}} \rightarrow F \rightarrow 0
$$

in $[\mathscr{C}, \mathrm{Ab}]$.

Recall that, for $\mathscr{A}$ and $\mathscr{B}$ categories, a functor $u_{2}: \mathscr{B} \rightarrow \mathscr{A}$ is said to be adjoint to a functor $u_{1}: \mathscr{A} \rightarrow \mathscr{B}$ (or equivalently $u_{1}$ is co-adjoint to $u_{2}$ ) if for any objects $F$ of $\mathscr{A}$ and $G$ of $\mathscr{B}$ there is an isomorphism

$$
\mathscr{A}\left(u_{2} G, F\right) \simeq \mathscr{B}\left(G, u_{1} F\right)
$$

which is natural in $F$ and $G$.

Proposition 1.3. For each bifunctor $U: \mathscr{C}_{1}^{\circ} \times \mathscr{C}_{2} \rightarrow \mathrm{Ab}$ the functor

$$
\otimes \mathscr{C}_{1} U:\left[\mathscr{C}_{1}, \mathrm{Ab}\right] \rightarrow\left[\mathscr{C}_{2}, \mathrm{Ab}\right]
$$

is adjoint to $[U,]_{\mathcal{B}_{2}}$. 
Proof. For each $G: \mathscr{C}_{2} \rightarrow \mathrm{Ab}$ the functors $\left[\otimes_{\mathscr{C}_{1}} U, G\right]$ and $\left[,[U, G]_{\mathscr{C}_{2}}\right]$ : $\left[\mathscr{C}_{1}, \mathrm{Ab}\right]^{\circ} \rightarrow \mathrm{Ab}$, given by $\left[\otimes_{\mathscr{C}_{1}} U, G\right](F)=\left[F \otimes_{\mathscr{C}_{1}} U, G\right]$ and $\left[\quad[U, G]_{\mathscr{C}_{2}}\right](F)$ $=\left[F,[U, G]_{\mathscr{C}_{2}}\right]$ for $F: \mathscr{C}_{1} \rightarrow \mathrm{Ab}$, are left continuous and have isomorphic values on representable functors, i.e., for $A$ in $\mathscr{C}_{1},\left[h^{A} \otimes_{\mathscr{C}_{1}} U, G\right] \simeq[U(A), G,] \simeq\left[h^{A},[U, G]_{\mathscr{C}_{2}}\right]$ by the Yoneda lemma. Thus using the exact sequence $\left(^{*}\right)$ we see that for each $F: \mathscr{C}_{1} \rightarrow \mathrm{Ab},\left[F \otimes_{\mathscr{C}_{1}} U, G\right] \simeq\left[F,[U, G]_{\mathscr{C}_{2}}\right]$ and this isomorphism is natural in $F$ and $G$.

2. Representing adjoint pairs. Let $\mathscr{A}$ and $\mathscr{B}$ be categories and let $u_{1}: \mathscr{A} \rightarrow \mathscr{B}$ and $u_{2}: \mathscr{B} \rightarrow \mathscr{A}$ be functors. We call $\left(u_{1}, u_{2}\right)$ an adjoint pair from $\mathscr{A}$ to $\mathscr{B}$ if $u_{2}$ is adjoint to $u_{1}$.

Given two adjoint pairs $\left(u_{1}, u_{2}\right)$ and $\left(v_{1}, v_{2}\right)$ from $\mathscr{A}$ to $\mathscr{B}$, we define a morphism of adjoint pairs $\alpha:\left(u_{1}, u_{2}\right) \rightarrow\left(v_{1}, v_{2}\right)$ by $\alpha=\left(\alpha_{1}, \alpha_{2}\right)$, where $\alpha_{1}$ and $\alpha_{2}$ are natural transformations $\alpha_{1}: v_{1} \rightarrow u_{1}$ and $\alpha_{2}: u_{2} \rightarrow v_{2}$ so that for all objects $F$ of $\mathscr{A}$ and $G$ of $\mathscr{B}$, the diagram

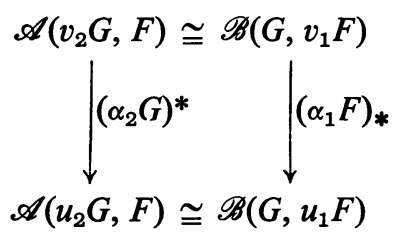

commutes.

Given two morphisms of adjoint pairs from $\mathscr{A}$ to $\mathscr{B}, \alpha=\left(\alpha_{1}, \alpha_{2}\right):\left(u_{1}, u_{2}\right) \rightarrow\left(v_{1}, v_{2}\right)$ and $\beta=\left(\beta_{1}, \beta_{2}\right):\left(v_{1}, v_{2}\right) \rightarrow\left(w_{1}, w_{2}\right)$, we define their composition by $\beta \circ \alpha=$ $\left(\alpha_{1} \circ \beta_{1}, \beta_{2} \circ \alpha_{2}\right):\left(u_{1}, u_{2}\right) \rightarrow\left(w_{1}, w_{2}\right)$, which is easily seen to be a morphism of adjoint pairs. We may therefore define a category $\operatorname{Adj}(\mathscr{A}, \mathscr{B})$ whose objects are adjoint pairs from $\mathscr{A}$ to $\mathscr{B}$ and whose morphisms and composition are defined above.

It is well known that

(1) Given an object $\left(u_{1}, u_{2}\right)$ of $\operatorname{Adj}(\mathscr{A}, \mathscr{B}), u_{2}$ is determined up to an isomorphism of functors by $u_{1}$ and conversely, and given a morphism $\alpha=\left(\alpha_{1}, \alpha_{2}\right)$ of $\operatorname{Adj}(\mathscr{A}, \mathscr{B})$, $\alpha_{2}$ is determined by $\alpha_{1}$ and conversely.

(2) For objects $\left(u_{1}, u_{2}\right)$ of $\operatorname{Adj}(\mathscr{A}, \mathscr{B})$ and $\left(u_{1}^{\prime}, u_{2}^{\prime}\right)$ of $\left.\operatorname{Adj}(\mathscr{B}, \mathscr{B})^{\prime}\right),\left(u_{1}^{\prime} \circ u_{1}, u_{2} \circ u_{2}^{\prime}\right)$ is an object of $\operatorname{Adj}\left(\mathscr{A}, \mathscr{B}^{\prime}\right)$ which is called the composition of $\left(u_{1}, u_{2}\right)$ and $\left(u_{1}^{\prime}, u_{2}^{\prime}\right)$.

(3) Given an object $\left(u_{1}, u_{2}\right)$ of $\operatorname{Adj}(\mathscr{A}, \mathscr{B}), u_{1}$ is left continuous and $u_{2}$ is right continuous.

It follows from 1.3 that for any two categories $\mathscr{C}_{1}$ and $\mathscr{C}_{2}$ we have a functor

$$
\Phi:\left[\mathscr{C}_{1}^{\circ} \times \mathscr{C}_{2}, \mathrm{Ab}\right] \rightarrow \operatorname{Adj}\left(\left[\mathscr{C}_{2}, \mathrm{Ab}\right],\left[\mathscr{C}_{1}, \mathrm{Ab}\right]\right)
$$

given by $\Phi(U)=\left([U,]_{\mathscr{C}_{2}}, \quad \otimes_{\mathscr{C}_{1}} U\right)$ for any bifunctor $U: \mathscr{C}_{1}^{\circ} \times \mathscr{C}_{2} \rightarrow \mathrm{Ab}$.

The following theorem and corollary are easy consequences of Proposition 9.1 of [1]. We give a simple proof that generalizes the proof for the case of modules. 
THEOREM 2.1. If $u:\left[\mathscr{C}_{1}, \mathrm{Ab}\right] \rightarrow\left[\mathscr{C}_{2}, \mathrm{Ab}\right]$ is right continuous, then there is a bifunctor $U: \mathscr{C}_{1}^{\circ} \times \mathscr{C}_{2} \rightarrow \mathrm{Ab}$ such that $u \cong \otimes_{\mathscr{C}_{1}} U$.

Proof. Let $h: \mathscr{C}_{1}^{\circ} \rightarrow\left[\mathscr{C}_{1}, \mathrm{Ab}\right]$ denote the functor such that $h(A)=h_{\mathscr{C}_{1}}^{A}\left(=h^{A}\right)$ for any object $A$ of $\mathscr{C}_{1}$. Then $u \circ h$ is an object of $\left[\mathscr{C}_{1}^{\circ},\left[\mathscr{C}_{2}, \mathrm{Ab}\right]\right]$. There is a canonical isomorphism

$$
\tau:\left[\mathscr{C}_{1}^{\circ},\left[\mathscr{C}_{2}, \mathrm{Ab}\right]\right] \cong\left[\mathscr{C}_{1}^{\circ} \times \mathscr{C}_{2}, \mathrm{Ab}\right]
$$

given by $\tau(t)(A, B)=t(A)(B)$ for all functors $t: \mathscr{C}_{1}^{\circ} \rightarrow\left[\mathscr{C}_{2}, \mathrm{Ab}\right]$ and for all objects $A$ of $\mathscr{C}_{1}$ and $B$ of $\mathscr{C}_{2}$. Let $U=\tau(u \circ h)$. Since, for each object $A$ of $\mathscr{C}_{1}$, we have an isomorphism $\varphi_{A}^{\prime}: U(A,) \rightarrow u\left(h^{A}\right)$ defined by the equalities

$$
U(A,)=\tau(u \circ h)(A,)=(u \circ h)(A)=u\left(h^{A}\right),
$$

and $u$ is right continuous, it follows from 1.2 that there is an isomorphism $\varphi: \otimes_{\mathscr{C}_{1}} U \cong u$.

It follows from this theorem and our remarks about adjoint functors that

Corollary 2.2. $\Phi:\left[\mathscr{C}_{1}^{\circ} \times \mathscr{C}_{2}, \mathrm{Ab}\right] \rightarrow \operatorname{Adj}\left(\left[\mathscr{C}_{2}, \mathrm{Ab}\right],\left[\mathscr{C}_{1}, \mathrm{Ab}\right]\right)$ is an equivalence of categories.

Therefore, if $\left(u_{1}, u_{2}\right)$ is an adjoint pair in $\operatorname{Adj}\left(\left[\mathscr{C}_{2}, \mathrm{Ab}\right],\left[\mathscr{C}_{1}, \mathrm{Ab}\right]\right)$, and $U: \mathscr{C}_{1}^{\circ} \times \mathscr{C}_{2}$ $\rightarrow \mathrm{Ab}$ is a bifunctor such that $\Phi(U) \cong\left(u_{1}, u_{2}\right)$, then $U$ is unique up to a canonical isomorphism. We shall say that $U$ represents the adjoint pair $\left(u_{1}, u_{2}\right)$, and that $U$ represents $u_{1}$ on the left and $U$ represents $u_{2}$ on the right.

As a trivial example, consider a category $\mathscr{C}$ and the adjoint pair $(I, I)$ from $[\mathscr{C}, \mathrm{Ab}]$ to itself, where $I$ is the identity functor on $[\mathscr{C}, \mathrm{Ab}]$. Then, if we let $h_{\mathscr{C}}: \mathscr{C}^{\circ} \times \mathscr{C}$ $\rightarrow \mathrm{Ab}$ denote the bifunctor $h_{\mathscr{C}}\left(A_{1}, A_{2}\right)=\mathscr{C}\left(A_{1}, A_{2}\right)$ for all objects $A_{1}$ and $A_{2}$ of $\mathscr{C}$, $h_{\mathscr{C}}$ represents $(I, I)$. That $h_{\mathscr{C}}$ represents $I$ on the left is a restatement of the "Yoneda lemma" and that $h_{\mathscr{C}}$ represents $I$ on the right is a restatement of 1.1(a).

Suppose we have an adjoint pair $\left(u_{1}, u_{2}\right)$ from $\left[\mathscr{C}_{2}, \mathrm{Ab}\right]$ to $\left[\mathscr{C}_{1}, \mathrm{Ab}\right]$ represented by a bifunctor $U: \mathscr{C}_{1}^{\circ} \times \mathscr{C}_{2} \rightarrow \mathrm{Ab}$, and an adjoint pair $\left(v_{1}, v_{2}\right)$ from $\left[\mathscr{C}_{3}, \mathrm{Ab}\right]$ to $\left[\mathscr{C}_{2}, \mathrm{Ab}\right]$ represented by a bifunctor $V: \mathscr{C}_{2}^{\circ} \times \mathscr{C}_{3} \rightarrow \mathrm{Ab}$. The composition of these adjoint pairs is an adjoint pair $\left(u_{1} \circ v_{1}, v_{2} \circ u_{2}\right)$ from $\left[\mathscr{C}_{3}, \mathrm{Ab}\right]$ to $\left[\mathscr{C}_{1}, \mathrm{Ab}\right]$.

We may define a bifunctor $U \otimes_{\mathscr{C}_{2}} V: \mathscr{C}_{1}^{\circ} \times \mathscr{C}_{3} \rightarrow \mathrm{Ab}$ by $U \otimes_{\mathscr{C}_{2}} V(A, C)=$ $U(A,) \otimes_{\mathscr{C}_{2}} V(, C)$ for all objects $A$ of $\mathscr{C}_{1}$ and $C$ of $\mathscr{C}_{3}$. Furthermore, if $F: \mathscr{C}_{1} \rightarrow \mathrm{Ab}$ is a functor, it follows from the associativity of the tensor product (see [4]) that $\left(F \otimes_{\mathscr{C}_{1}} U\right) \otimes_{\mathscr{C}_{2}} V \cong F \otimes_{\mathscr{C}_{1}}\left(U \otimes_{\mathscr{C}_{2}} V\right)$. As an easy consequence, we have

Proposition 2.3. Let $\left(u_{1}, u_{2}\right)$ and $\left(v_{1}, v_{2}\right)$ be adjoint pairs represented by $U: \mathscr{C}_{1}^{\circ} \times \mathscr{C}_{2} \rightarrow \mathrm{Ab}$ and $V: \mathscr{C}_{2}^{\circ} \times \mathscr{C}_{3} \rightarrow \mathrm{Ab}$, respectively. Then the adjoint pair $\left(u_{1} \circ v_{1}, v_{2} \circ u_{2}\right)$ is represented by $U \otimes \mathscr{C}_{2} V$.

Since for any functor $F: \mathscr{C}_{3} \rightarrow \mathrm{Ab}, u_{1} \circ v_{1}(F) \cong\left[U,[V, F]_{\mathscr{C}_{3}}\right] \mathscr{C}_{2}$, and left adjoints of a functor are canonically isomorphic, we have 
Corollary 2.4. For any bifunctors $U: \mathscr{C}_{1}^{\circ} \times \mathscr{C}_{2} \rightarrow \mathrm{Ab}$ and $V: \mathscr{C}_{2}^{\circ} \times \mathscr{C}_{3} \rightarrow \mathrm{Ab}$ and for any functor $F: \mathscr{C}_{3} \rightarrow \mathrm{Ab}$, there is a canonical isomorphism

$$
\left[U \otimes_{\mathscr{C}_{2}} V, F\right]_{\mathscr{C}_{3}} \cong\left[U,[V, F]_{\mathscr{C}_{3}}\right]_{\mathscr{C}_{2}}
$$

natural in $U, V$, and $F$.

3. Satellites. In this section, $\mathscr{C}$ and $\mathscr{D}$ will be abelian categories.

For a functor $F: \mathscr{C} \rightarrow \mathrm{Ab}$, there are functors $S^{n} F$ and $S_{n} F$ for each integer $n \geqq 0$, the $n$th right and left satellites of $F$ respectively (which are defined below). We may consider $S^{n}$ and $S_{n}$ as functors from [ $\left.\mathscr{C}, \mathrm{Ab}\right]$ to itself. The result of this section is that $\left(S_{n}, S^{n}\right)$ is an adjoint pair which is represented by the bifunctor $\operatorname{Ext}_{\mathscr{C}}^{n}():, \mathscr{C}^{\circ} \times \mathscr{C} \rightarrow \mathrm{Ab}$.

Let $F_{1}$ and $F_{2}$ be functors from a category $\mathscr{C}$ to a category $\mathscr{D}$. Then $F_{1}$ and $F_{2}$ are connected if for every short exact sequence $E: 0 \rightarrow A^{\prime} \rightarrow A \rightarrow A^{\prime \prime} \rightarrow 0$ in $\mathscr{C}$ there is a map $\Delta_{E}: F_{1}\left(A^{\prime \prime}\right) \rightarrow F_{2}\left(A^{\prime}\right)$ natural in $E$ so that the complex $F_{1}\left(A^{\prime}\right) \rightarrow F_{1}(A)$ $\rightarrow F_{1}\left(A^{\prime \prime}\right) \rightarrow F_{2}\left(A^{\prime}\right) \rightarrow F_{2}(A) \rightarrow F_{2}\left(A^{\prime \prime}\right)$ is of order 2 (that is, composition of two successive morphisms is the zero morphism). We shall indicate that $F_{1}$ and $F_{2}$ are connected by $\Delta$, by the triple $\left(F_{1}, \Delta, F_{2}\right)$, which we call a connected triple in $[\mathscr{C}, \mathscr{D}]$.

Given two connected triples $\left(F_{1}, \Delta, F_{2}\right)$ and $\left(G_{1}, \Delta^{\prime}, G_{2}\right)$, we may define a morphism of connected triples to be a pair $\left(\alpha_{1}, \alpha_{2}\right):\left(F_{1}, \Delta, F_{2}\right) \rightarrow\left(G_{1}, \Delta^{\prime}, G_{2}\right)$, where $\alpha_{1}: F_{1} \rightarrow G_{1}$ and $\alpha_{2}: F_{2} \rightarrow G_{2}$ are natural transformations such that, for every exact sequence $E: 0 \rightarrow A^{\prime} \rightarrow A \rightarrow A^{\prime \prime} \rightarrow 0$, we have $\Delta_{E}^{\prime} \circ \alpha_{1}\left(A^{\prime \prime}\right)=\alpha_{2}\left(A^{\prime}\right) \circ \Delta_{E}$.

If $\left(\alpha_{1}, \alpha_{2}\right):\left(F_{1}, \Delta, F_{2}\right) \rightarrow\left(G_{1}, \Delta^{\prime}, G_{2}\right)$ and $\left(\beta_{1}, \beta_{2}\right):\left(G_{1}, \Delta^{\prime}, G_{2}\right) \rightarrow\left(H_{1}, \Delta^{\prime \prime}, H_{2}\right)$ are morphisms of connected triples, then the composition $\left(\beta_{1}, \beta_{2}\right) \circ\left(\alpha_{1}, \alpha_{2}\right)=\left(\beta_{1} \circ \alpha_{1}\right.$, $\left.\beta_{2} \circ \alpha_{2}\right)$ is also. Consequently, we may define a category $\Delta[\mathscr{C}, \mathscr{D}]$ whose objects are connected triples in $[\mathscr{C}, \mathscr{D}]$ and whose morphisms and composition are defined above. We have two functors $\pi_{i}: \Delta[\mathscr{C}, \mathscr{D}] \rightarrow[\mathscr{C}, \mathscr{D}]$ defined by $\pi_{i}\left(F_{1}, \Delta, F_{2}\right)=F_{i}$, $i=1,2$, for any connected triple $\left(F_{1}, \Delta, F_{2}\right)$ in $[\mathscr{C}, \mathscr{D}]$.

Let $F: \mathscr{C} \rightarrow \mathscr{D}$ be a functor. Then $F$ has a right satellite if there is a functor $S^{1} F: \mathscr{C} \rightarrow \mathscr{D}$ so that $F$ and $S^{1} F$ are connected (via $\Delta$, let us say), and given any connected triple $\left(G_{1}, \Delta^{\prime}, G_{2}\right)$ in $[\mathscr{C}, \mathscr{D}]$ and any natural transformation $\alpha: F \rightarrow G_{1}$, there is a unique natural transformation $\alpha^{\prime}: S^{1} F \rightarrow G_{2}$ so that $\left(\alpha, \alpha^{\prime}\right):\left(F, \Delta, S^{1} F\right)$ $\rightarrow\left(G_{1}, \Delta^{\prime}, G_{2}\right)$ is a morphism of connected triples. Similarly, $F$ has a left satellite if there is a functor $S_{1} F: \mathscr{C} \rightarrow \mathscr{D}$ so that $S_{1} F$ and $F$ are connected (by $\Delta$ ), and given any connected triple $\left(G_{1}, \Delta^{\prime}, G_{2}\right)$ and a natural transformation $\alpha: G_{2} \rightarrow F$, there is a unique natural transformation $\alpha^{\prime}: G_{1} \rightarrow S_{1} F$ so that $\left(\alpha^{\prime}, \alpha\right):\left(G_{1}, \Delta^{\prime}, G_{2}\right) \rightarrow$ $\left(S_{1} F, \Delta, F\right)$ is a morphism of connected pairs.

We shall denote the connected triples $\left(F, \Delta, S^{1} F\right)$ and $\left(S_{1} F, \Delta, F\right)$ by $\Delta S^{1}(F)$ and $\Delta S_{1}(F)$ respectively. If $[\mathscr{C}, \mathscr{D}]$ has right satellites (that is, all functors $F: \mathscr{C} \rightarrow \mathscr{D}$ have right satellites), then $\Delta S^{1}$ defines a functor $\Delta S^{1}:[\mathscr{C}, \mathscr{D}] \rightarrow \Delta[\mathscr{C}, \mathscr{D}]$, and similarly if $[\mathscr{C}, \mathscr{D}]$ has left satellites, $\Delta S_{1}$ defines a functor $\Delta S_{1}:[\mathscr{C}, \mathscr{D}] \rightarrow \Delta[\mathscr{C}, \mathscr{D}]$. In fact, we may translate the definition of $\dot{\Delta S^{1}}$ and $\Delta S_{1}$ into the following: 
Proposition 3.1. If $[\mathscr{C}, \mathscr{D}]$ has right satellites, then $\Delta S^{1}$ is the adjoint of $\pi_{1}$. If $[\mathscr{C}, \mathscr{D}]$ has left satellites, then $\Delta S_{1}$ is the co-adjoint of $\pi_{2}$.

Suppose $[\mathscr{C}, \mathscr{D}]$ has satellites (both right and left). Then we have adjoint pairs $\left(\Delta S_{1}, \pi_{2}\right)$ from $[\mathscr{C}, \mathscr{D}]$ to $\Delta[\mathscr{C}, \mathscr{D}]$ and $\left(\pi_{1}, \Delta S^{1}\right)$ from $\Delta[\mathscr{C}, \mathscr{D}]$ to $[\mathscr{C}, \mathscr{D}]$, and composing gives us an adjoint pair $\left(\pi_{1} \circ \Delta S_{1}, \pi_{2} \circ \Delta S^{1}\right)$ from [ $\left.\mathscr{C}, \mathscr{D}\right]$ to itself. But $\pi_{1} \circ \Delta S_{1}(F)=S_{1} F$ and $\pi_{2} \circ \Delta S^{1}(F)=S^{1} F$, which proves the following:

Corollary 3.2( $\left(^{1}\right)$. If $[\mathscr{C}, \mathscr{D}]$ has satellites, then $S^{1}:[\mathscr{C}, \mathscr{D}] \rightarrow[\mathscr{C}, \mathscr{D}]$ is adjoint to $S_{1}$.

If $F: \mathscr{C} \rightarrow \mathscr{D}$ is a functor, we define, in the usual way, the higher satellites of $F$ as follows:

$$
\begin{array}{lll}
S^{0} F=F, & S^{n+1} F=S^{1}\left(S^{n} F\right) & \text { for all } n \geqq 0, \\
S_{0} F=F, & S_{n+1} F=S_{1}\left(S_{n} F\right) & \text { for all } n \geqq 0 .
\end{array}
$$

COROLlary 3.3. If $[\mathscr{C}, \mathscr{D}]$ has satellites, then $S^{n}$ is adjoint to $S_{n}$ for all $n \geqq 0$.

The proof is by induction, using the fact that composition of adjoint pairs is an adjoint pair.

If $\mathscr{D}=\mathrm{Ab}$, then $[\mathscr{C}, \mathrm{Ab}]$ has satellites for any abelian category $\mathscr{C}$ (see [2]). To compute the bifunctor representing the adjoint pair $\left(S_{n}, S^{n}\right)$, we may use the proof of Theorem 2.1. Since for any object $A$ of $\mathscr{C}$, it is known that $S^{n}\left(h_{\mathscr{C}}^{A}\right) \cong \operatorname{Ext}_{\mathscr{C}}^{n}(A, \quad)$, we have the following:

THEOREM 3.4. For all integers $n \geqq 0$, the functors $S_{n}$ and $S^{n}$ from $[\mathscr{C}, \mathrm{Ab}]$ to itself form an adjoint pair $\left(S_{n}, S^{n}\right)$ which is represented by the bifunctor $\operatorname{Ext}_{\mathscr{C}}^{n}(, \quad)$.

We remark that the fact that $S_{n}$ is represented on the left by $\operatorname{Ext}_{\mathscr{G}}^{n}($,$) is a$ result of Yoneda [7], while the fact that $S^{n}$ is represented on the right by $\operatorname{Ext}_{\mathscr{C}}^{n}(, \quad)$ is a result in [4].

4. Duality and adjoints of induced functors. In this section we define, for a function $F: \mathscr{C} \rightarrow \mathrm{Ab}$, its dual $F^{*}: \mathscr{C}^{\circ} \rightarrow \mathrm{Ab}$ and prove some theorems concerning $F^{*}$. Using these results, we are able to prove some results about a bifunctor $U$ when $U$ represents an adjoint pair $\left(u_{1}, u_{2}\right)$ from $\left[\mathscr{C}_{2}, \mathrm{Ab}\right]$ to $\left[\mathscr{C}_{1}, \mathrm{Ab}\right]$ with $u_{1}$ exact and continuous. In particular, we deal with the case where $u_{1}$ is induced by a functor $\mathscr{C}_{1} \rightarrow \mathscr{C}_{2}$.

Let $V: \mathscr{C}_{1}^{\circ} \times \mathscr{C}_{2} \rightarrow \mathrm{Ab}$ be a bifunctor. Then we define a functor $H_{\mathscr{C}_{2}}[, V]$ : $\left[\mathscr{C}_{2}, \mathrm{Ab}\right]^{\circ} \rightarrow\left[\mathscr{C}_{1}^{\circ}, \mathrm{Ab}\right]$ as follows: for a functor $F: \mathscr{C}_{2} \rightarrow \mathrm{Ab}$ and an object $A$ of $\mathscr{C}_{1}$, we let $H_{\mathscr{C}_{2}}[F, V](A)=[F, V(A)$,$] . Similarly, we define a functor$

$$
H^{\mathscr{C}_{1}}[, V]:\left[\mathscr{C}_{1}^{\circ}, \mathrm{Ab}\right]^{\circ} \rightarrow\left[\mathscr{C}_{2}, \mathrm{Ab}\right]
$$

(1) We thank our colleague, Paul Palmquist, for suggesting using universal properties of satellites to prove this theorem. 
as follows: for a functor $G: \mathscr{C}_{1}^{\circ} \rightarrow \mathrm{Ab}$ and an object $B$ of $\mathscr{C}_{2}, H^{\mathscr{C}_{1}}[G, V](B)=$ $[G, V(, B)]$.

As a direct consequence of 1.2 we have

Proposition 4.1. Let $F: \mathscr{C} \rightarrow \mathrm{Ab}$ be a functor and let $F^{*}=H_{\mathscr{C}}\left[F, h_{\mathscr{C}}\right]$. Then there exists a unique natural transformation

$$
\beta(, F): \quad \otimes_{\mathscr{C}} F^{*} \rightarrow[F,]
$$

of functors from $[\mathscr{C}, \mathrm{Ab}]$ to $\mathrm{Ab}$, natural in $F$, such that $\beta\left(h^{A}, F\right)$ is the composition of isomorphisms

$$
h^{A} \otimes F^{*} \simeq F^{*}(A)=H_{\mathscr{C}}\left[F, h_{\mathscr{C}}\right](A)=\left[F, h^{A}\right]
$$

Recall that, for an object $P$ of an abelian category $\mathscr{A}, P$ is a small projective if and only if $h_{\mathscr{A}}^{P}$ is right continuous. In particular, it follows that an object $F$ in $[\mathscr{C}, \mathrm{Ab}]$ is a small projective if and only if there is a finite collection of objects $\left(B_{i}\right)_{I}$ of $\mathscr{C}$ such that $F$ is a direct summand of $\sum_{I} h_{\mathscr{C}}^{B_{i}}$.

Proposition 4.2. For $F: \mathscr{C} \rightarrow \mathrm{Ab}$ the following are equivalent:

(a) $\beta(, F): \otimes_{\mathscr{C}} F^{*} \rightarrow[F$,$] is a natural equivalence.$

(b) $\beta(G, F)$ is an epimorphism for each $G: \mathscr{C} \rightarrow \mathrm{Ab}$.

(c) $\beta(F, F)$ is an epimorphism.

(d) $F$ is a small projective in $[\mathscr{C}, \mathrm{Ab}]$.

Proof. $a \Rightarrow b \Rightarrow c$ is trivial.

$d \Rightarrow a$ is a direct consequence of the definition of small projective and 1.2.

$c \Rightarrow d$. We know that there exists a collection $\left(A_{j}\right)_{J}$ of objects in $\mathscr{C}$ and transformations $\left\{s_{j}: h_{\mathscr{E}}^{A}, \rightarrow F\right\}$ such that $\sum s_{j}: \sum_{j \in J} h_{\mathscr{E}}^{A} \rightarrow F$ is an epimorphism. Since

$\otimes F^{*}$ is right continuous we have the commutative diagram

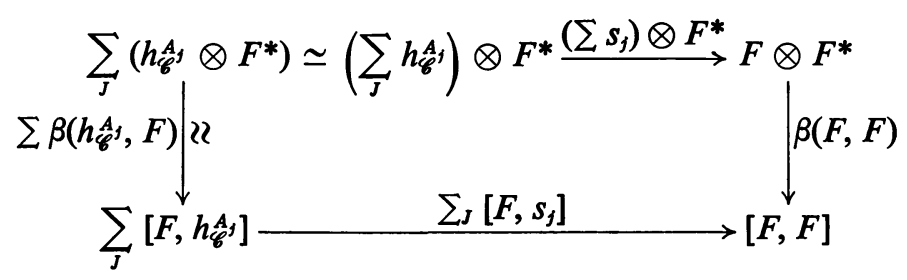

with the top map an epimorphism. Thus $\beta(F, F)$ is an epimorphism implies $\sum_{J}\left[F, s_{j}\right]$ is an epimorphism and hence the existence of a finite subset $I$ of $J$ and elements $t_{i} \in\left[F, h_{\mathscr{C}}^{A}\right], i \in I$, such that

$$
\left(F \stackrel{\sum t_{i}}{\longrightarrow} \sum_{I} h_{\mathscr{C}}^{A} \stackrel{\sum s_{i}}{\longrightarrow} F\right)=1_{F} .
$$

Thus by the remark preceding this proposition $F$ is a small projective. 
Let $F: \mathscr{C} \rightarrow \mathrm{Ab}$, and let $F^{* *}$ denote the functor $H^{\mathscr{C}}\left[H_{\mathscr{C}}[F, h], h\right]$. Let $A$ and $B$ be objects of $\mathscr{C}$. Then we have a map

$$
\nu_{F}(A, B): F(B) \rightarrow \operatorname{Hom}(\operatorname{Hom}(F(B), \mathscr{C}(A, B)), \mathscr{C}(A, B))
$$

given by $\nu_{F}(A, B)(x)(\lambda)=\lambda(x)$ for all $x$ in $F(B)$ and $\lambda \in \operatorname{Hom}(F(B), \mathscr{C}(A, B))$. It is easy to show that this defines a natural transformation $\nu_{F}: F \rightarrow F^{* *}$ which is natural in $F$.

Proposition 4.3. If $F$ is a small projective in $[\mathscr{C}, \mathrm{Ab}]$, then $\nu_{F}: F \rightarrow F^{* *}$ is an isomorphism.

Proof. It is easy to check that for each object $A$ of $\mathscr{C}, h^{A *} \cong h_{A}$ and $h^{A * *} \cong h^{A}$, and that $\nu_{h}^{A}$ is this isomorphism. Therefore, by a direct sum argument, $\nu_{F}$ is an isomorphism for any small projective $F$.

We now generalize these results to bifunctors. Let $\mathscr{C}_{1}, \mathscr{C}_{2}$, and $\mathscr{C}_{3}$ be categories. Given bifunctors $U: \mathscr{C}_{3}^{\circ} \times \mathscr{C}_{2} \rightarrow \mathrm{Ab}$ and $V: \mathscr{C}_{1}^{\circ} \times \mathscr{C}_{2} \rightarrow \mathrm{Ab}$, we define a bifunctor $H_{\mathscr{C}_{2}}[U, V](A, B)=[U(B),, V(A)$,$] for any objects A$ of $\mathscr{C}_{1}$ and $B$ of $\mathscr{C}_{3}$. If $W: \mathscr{C}_{1}^{\circ} \times \mathscr{C}_{3} \rightarrow \mathrm{Ab}$ is a bifunctor and $V$ is as above, we define a bifunctor

$$
H^{\mathscr{C}_{1}}[W, V]: \mathscr{C}_{3}^{\circ} \times \mathscr{C}_{2} \rightarrow \mathrm{Ab}
$$

by $H^{\mathscr{C}_{1}}[W, V](C, B)=[W(, C), V(, B)]$ for any objects $B$ of $\mathscr{C}_{2}$ and $C$ of $\mathscr{C}_{3}$.

Proposition 4.4. Let $U: \mathscr{C}_{1}^{\circ} \times \mathscr{C}_{2} \rightarrow \mathrm{Ab}$ be a bifunctor. Then

(a) there is a natural transformation

$$
\beta_{U}: \quad \otimes_{\mathscr{C}_{2}} H_{\mathscr{C}_{2}}\left[U, h_{\mathscr{C}_{2}}\right] \rightarrow[U,]_{\mathscr{C}_{2}}
$$

of functors from $\left[\mathscr{C}_{2}, \mathrm{Ab}\right]$ to $\left[\mathscr{C}_{1}, \mathrm{Ab}\right]$ such that $\beta_{U}$ is an equivalence of functors if and only if $U(A, \quad)$ is a small projective of $\left[\mathscr{C}_{2}, \mathrm{Ab}\right]$ for every $A$ in $\mathscr{C}_{1}$, and

(b) there is a natural transformation of bifunctors $\nu_{U}: U \rightarrow H^{\mathscr{C}_{2}}\left[H_{\mathscr{C}_{2}}\left[U, h_{\mathscr{C}_{2}}\right], h_{\mathscr{C}_{2}}\right]$ such that if $U(A, \quad)$ is a small projective of $\left[\mathscr{C}_{2}, \mathrm{Ab}\right]$ for every $A$ in $\mathscr{C}_{1}$, then $\nu_{U}$ is an isomorphism.

Proof. (a) For each $G: \mathscr{C}_{2} \rightarrow \mathrm{Ab}$ and $A$ in $\mathscr{C}_{1}$, we let $\beta_{U}(G)(A)$ equal $\beta(G, U(A, \quad))$ defined in 4.1. The remainder of (a) follows from 4.2.

(b) For each $A$ in $\mathscr{C}_{1}$, we let $\nu_{U}(A, \quad)$ be the natural transformation

$$
\nu_{U(A,)}: U(A,) \rightarrow U(A, \quad)^{* *}=H^{\mathscr{C}_{2}}\left[H_{\mathscr{C}_{2}}\left[U, h_{\mathscr{C}_{2}}\right], h_{\mathscr{C}_{2}}\right](A, \quad)
$$

defined above in 4.3. By 4.3 if $U(A, \quad)$ is a small projective for every $A$ in $\mathscr{C}_{1}$ then $\nu_{U}$ is an equivalence of functors.

Let $\mathscr{C}_{1}$ and $\mathscr{C}_{2}$ be two categories, $\left(u_{1}, u_{2}\right)$ an adjoint pair from [ $\mathscr{C}_{2}, \mathrm{Ab}$ ] to [ $\mathscr{C}_{1}, \mathrm{Ab}$ ], and $U: \mathscr{C}_{1}^{\circ} \times \mathscr{C}_{2} \rightarrow \mathrm{Ab}$ the bifunctor representing $\left(u_{1}, u_{2}\right)$.

$u_{1}$ is an exact continuous functor if and only if $u_{1} \cong[U,]_{\mathscr{C}_{2}}$ is right continuous. Since exactness and limits in $\left[\mathscr{C}_{1}, \mathrm{Ab}\right]$ are computed "pointwise," we see that $[U,]_{\mathscr{C}_{2}}$ is right continuous if and only if for each $A$ in $\mathscr{C}_{1}, U(A$,$) is a small$ projective in $\left[\mathscr{C}_{2}, \mathrm{Ab}\right]$. These remarks, and $4.4(\mathrm{a})$ give us the following: 
Proposition 4.5. Let $\left(u_{1}, u_{2}\right)$ be an adjoint pair from $\left[\mathscr{C}_{2}, \mathrm{Ab}\right]$ to $\left[\mathscr{C}_{1}, \mathrm{Ab}\right]$ represented by $U: \mathscr{C}_{1}^{\circ} \times \mathscr{C}_{2} \rightarrow \mathrm{Ab}$. Then the following statements are equivalent:

(a) $u_{1}$ is an exact continuous functor.

(b) $U(A, \quad)$ is a small projective for each object $A$ of $\mathscr{C}_{1}$.

(c) $u_{1}$ is represented on the right by $H_{\mathscr{C}_{2}}\left[U, h_{\mathscr{C}_{2}}\right]$, i.e., $u_{1} \simeq \otimes_{\mathscr{C}_{2}} H_{\mathscr{C}_{2}}\left[U, h_{\mathscr{C}_{2}}\right]$.

For example, suppose $u_{1}=f^{*}:\left[\mathscr{C}_{2}, \mathrm{Ab}\right] \rightarrow\left[\mathscr{C}_{1}, \mathrm{Ab}\right]$, where $f: \mathscr{C}_{1} \rightarrow \mathscr{C}_{2}$ is a functor. Then, since $f^{*}$ is exact and continuous, $f^{*}$ is represented on the left by a bifunctor $U: \mathscr{C}_{1}^{\circ} \times \mathscr{C}_{2} \rightarrow \mathrm{Ab}$ such that for all objects $A$ of $\mathscr{C}_{1}, U(A, \quad)$ is a small projective in $\left[\mathscr{C}_{2}, \mathrm{Ab}\right]$. Since $f^{*}$ is represented on the right by $H_{\mathscr{C}_{2}}\left[U, h_{\mathscr{C}_{2}}\right]$, we can compute $H_{\mathscr{C}_{2}}\left[U, h_{\mathscr{C}_{2}}\right]$ by the method used in the proof of 2.1 with the result that

$$
H_{\mathscr{C}_{2}}\left[U, h_{\mathscr{C}_{2}}\right] \cong \mathscr{C}_{2}(, f) .
$$

Since, by $4.4(\mathrm{~b}), U \cong H^{\mathscr{C}_{2}}\left[H_{\mathscr{C}_{2}}\left[U, h_{\mathscr{C}_{2}}\right], h_{\mathscr{C}_{2}}\right]$, we can compute $U$, with the result that $U \cong \mathscr{C}_{2}(f$,$) . Therefore, it follows that$

THEOREM 4.6. Let $f: \mathscr{C}_{1} \rightarrow \mathscr{C}_{2}$ be a functor, let $f^{*}:\left[\mathscr{C}_{2}, \mathrm{Ab}\right] \rightarrow\left[\mathscr{C}_{1}, \mathrm{Ab}\right]$ be the induced functor, and let $f_{*}$ denote its adjoint. Then the adjoint pair $\left(f^{*}, f_{*}\right)$ is represented by the bifunctor $\mathscr{C}_{2}(f, \quad): \mathscr{C}_{1}^{\circ} \times \mathscr{C}_{2} \rightarrow \mathrm{Ab}$.

We can give a trivial proof of the following known result:

CoRollary 4.7. If $f, f^{*}$, and $f_{*}$ are as in 4.6 , and $f: \mathscr{C}_{1} \rightarrow \mathscr{C}_{2}$ is a full and faithful functor (for example, $\mathscr{C}_{1}$ a full subcategory of $\mathscr{C}_{2}$, and $f$ the inclusion functor), then $f^{*} \circ f_{*} \cong I$, the identity on $\left[\mathscr{C}_{1}, \mathrm{Ab}\right]$.

Proof. Since $f$ is full and faithful, we have $\mathscr{C}_{1}(,) \cong \mathscr{C}_{2}(f, f)$. Since $f_{*}$ is represented on the right by $\mathscr{C}_{2}(f, \quad)$ and $f^{*}$ is represented on the right by $\mathscr{C}_{2}(, f)$, we have that $f^{*} \circ f_{*}$ is represented on the right by $\mathscr{C}_{2}(f,) \otimes \mathscr{C}_{2} \mathscr{C}_{2}(, f)$ by Proposition 2.3.

Now, for all objects $A_{1}$ and $A_{2}$ of $\mathscr{C}_{1}$,

$$
\begin{aligned}
\mathscr{C}_{2}(f,) \otimes \mathscr{C}_{2} \mathscr{C}_{2}(, f)\left(A_{1}, A_{2}\right) & =\mathscr{C}_{2}\left(f\left(A_{1}\right), \quad\right) \otimes \mathscr{C}_{2} \mathscr{C}_{2}\left(, f\left(A_{2}\right)\right) \\
& =h_{\mathscr{C}_{2}}^{f\left(A_{1}\right)} \otimes \mathscr{C}_{2} \mathscr{C}_{2}\left(, f\left(A_{2}\right)\right) \cong \mathscr{C}_{2}\left(f\left(A_{1}\right), f\left(A_{2}\right)\right) \\
& \cong \mathscr{C}_{1}\left(A_{1}, A_{2}\right) ;
\end{aligned}
$$

so that $f^{*} \circ f_{*}$ is represented on the right by the bifunctor $\mathscr{C}_{1}()=,h_{\mathscr{C}_{1}}$, which also represents the identity functor on the right. The equivalence of categories given in 2.2 completes the proof.

\section{REFERENCES}

1. M. André, Categories of functors and adjoint functors, Amer. J. Math. 88 (1966), 529-543. MR 33 \#5693.

2. D. A. Buchsbaum, Satellites and universal functors, Ann. of Math. (2) 71 (1960), 199-209. MR 22 \#3751. 
3. S. Eilenberg, Abstract description of some basic functors, J. Indian Math. Soc. 24 (1960), 231-234. MR 23 \#A2454.

4. J. L. Fisher, The tensor product of functors; satellites; and derived functors, J. Algebra 8 (1968), 277-294. MR 38 \#5874.

5. D. M. Kan, Adjoint functors, Trans. Amer. Math. Soc. 87 (1958), 294-329. MR 24 \#A1301.

6. C. E. Watts, Intrinsic characterizations of some additive functors, Proc. Amer. Math. Soc. 11 (1960), 5-8. MR 22 \#9528.

7. N. Yoneda, On Ext and exact sequences, J. Fac. Sci. Univ. Tokyo Sect. I 8 (1960), 507-576. MR 37 \#1445.

UNIVERSITY OF CALIFORNIA,

IRVINE, CALIFORNIA 92664 